Supplement of Solid Earth, 10, 1409-1428, 2019

https://doi.org/10.5194/se-10-1409-2019-supplement

C Author(s) 2019. This work is distributed under

the Creative Commons Attribution 4.0 License.

(c) (1)

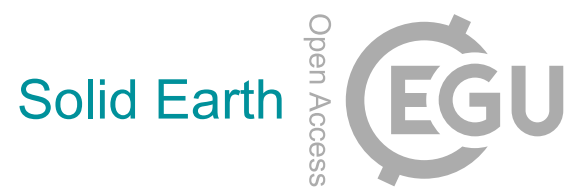

Supplement of

\title{
Chemical heterogeneities in the mantle: progress towards a general quantitative description
}

Massimiliano Tirone

Correspondence to: Massimiliano Tirone (max.tirone@gmail.com)

The copyright of individual parts of the supplement might differ from the CC BY 4.0 License. 


\section{S1 Supplementary Data}

This section describes the additional material available through an external data repository. The public link to access all the files is:

https://figshare.com/collections/G-KINB/4079111

List of the available files:

$$
\begin{aligned}
& \text { - TWOPD-G-KIN.DATA.ZIP } \\
& \text { - TWOPD-G-KIN.MOVIEI.AVI } \\
& \text { - TWOPD-G-KIN.MOVIE5.AVI } \\
& \text { - 2D-G-KIN.DATA.ZIP } \\
& \text { - 2D-G-KIN.MOVIE5A.AVI } \\
& \text { - 2D-G-KIN.MOVIE5B.AVI }
\end{aligned}
$$

\section{S1.1 1-D Simulations}

The zip file TWOPD-G-KIN.DATA. ZIP includes the data of three 1-D simulations assuming that the initial proportion of the two assemblages is 1:1, 5:1 and 50:1 $(f=1,5,50)$. The details of the models are discussed in the main text. For every simulation there are two data files: TWOPD-G-KIN1 . 1. DAT and TWOPD-G-KIN2 . 1. DAT for the case with 1:1 proportion, TWOPD-G-KIN1.5.DAT, TWOPD-G-KIN2.5.DAT and TWOPD-G-KIN1.50.DAT, TWOPD-G-KIN2.50.DAT for the models with initial proportion 5:1 and 50:1, respectively. The data files are divided in blocks, each block of data refers to a particular time step.

The first data file for each simulation (TWOPD-G-KIN1.1.DAT. TWOPD-G-KIN1.5.DAT and TWOPD-G-KIN1.50.DAT) includes in every block, distance, $G(*)$ (joules) and the grid step size for the two sub-systems. The number of grid points for sub-system $A$ and $B$ are 101 and 101 in the first simulation, 501 and 101 in the second simulation, 1001 and 101 in the third simulation. Time step is 4, 40 and 800 for the three simulations. Data are stored every 20, 20, 50 numerical time steps respectively. As discussed in the main text, time, distance and step size have arbitrary units.

The second data file of each simulation (TWOPD-G-KIN2.1.DAT, TWOPD-G-KIN2.5.DAT and TWOPD-G-KIN2 50 . DAT) includes in every block, distance and abundance of nine oxides (grams or wt $\%$ ) which describes the bulk composition at every grid point. The listed oxides are: $\mathrm{SiO}_{2}, \mathrm{TiO}_{2}, \mathrm{Al}_{2} \mathrm{O}_{3}, \mathrm{Fe}_{2} \mathrm{O}_{3}, \mathrm{Cr}_{2} \mathrm{O}_{3}, \mathrm{FeO}, \mathrm{MgO}, \mathrm{CaO}$ and $\mathrm{Na}_{2} \mathrm{O}$.

Two 1-D animations TWOPD-G-KIN. MOVIE1. AVI and TWOPD-G-KIN. MOVIE5. AVI are based on the simulations with $f=1$ and $f=5$. The relative data are included in the zip file TWOPD-G-KIN. DATA. ZIP.

\section{S1.2 2-D Simulations}

The results of two 2-D simulations are included in the zip file 2D-G-KIN. DATA. ZIP. For both simulations the initial proportion of the two assemblages is set to 5:1. The interface between the two sub-systems is a vertical line. The first simulation assumes that assemblage $A$ becomes mobile downwards at time $=1000000$ (arbitrary units), while in the second simulation the dynamic assemblage is $B$. The velocity of the moving assemblages is set to 0.00625 (arbitrary units). New material entering from the top side has the same bulk composition of the initial assemblage. The composition is reported in the main text and in the data files here below. Output data are stored every 400 time steps and the simulation time step is 16 (arbitrary units). Each block of data defined by the label "ZONE" provides information related to a particular time step.

The first data file of each simulation (2D-G-KIN1.5A. DAT and 2D-G-KIN1.5B.DAT) includes the distance x-direction, 
y-direction and $G(*)$. The number of grid points in the x-direction is 251 and 51 in sub-system $A$ and $B$, respectively (total initial distance is 500 and 100 in arbitrary units). The number of grid points in the y-direction is 51 (total distance is 50 in arbitrary units). A block of data is divided in sub-blocks. Each sub-block consists of $(251+51) \times 51$ data points. The first sub-block contains the x-coordinate defining the numerical grid, the second sub-block the y-coordinate and the third sub-block the $G(*)$ values at the corresponding grid points.

The second data file of each simulation (2D-G-KIN2 . 5A. DAT and 2D-G-KIN2 . 5B.DAT) follows the same data structure, except that instead of $G(*)$, nine bulk oxides are listed in nine sub-blocks. The sequence of oxides is the same reported for the 1-D models.

The data in the zip file 2D-G-KIN. DATA. AVI have been used to create two animations, 2D-G-KIN. MOVIE5A. AVI and 2D-G-KIN. MOVIE5B.AVI, both are available following the link to the external data repository.

\section{S2 Supplementary Table}

The following table reports the initial bulk composition and the proportion factor $f$ of the two sub-systems for all the 43 cases considered in this study (see sections 2.1 and 2.2 in the main text). 
Table S1. Initial bulk composition of the two assemblages and proportion factor $f$.

\begin{tabular}{|c|c|c|c|c|c|c|c|c|c|c|}
\hline bulk comp. & $\left(A_{0}\right)$ & $\left(B_{0}\right)$ & $\overline{\left(A_{0}\right)}$ & $\overline{\left(B_{0}\right)}$ & $\left(A_{0}\right)$ & $\left(B_{0}\right)$ & $\left(A_{0}\right)$ & $\left(B_{0}\right)$ & $\left(A_{0}\right)$ & $\left(B_{0}\right)$ \\
\hline oxides (g or wt $\%$ ) & \multicolumn{2}{|c|}{$\# 1(\mathrm{f}=1)$} & \multicolumn{2}{|c|}{$\# 2(\mathrm{f}=1.2)$} & \multicolumn{2}{|c|}{$\# 3(\mathrm{f}=1.3)$} & \multicolumn{2}{|c|}{$\# 4(\mathrm{f}=1.6)$} & \multicolumn{2}{|c|}{$\# 5(\mathrm{f}=2)$} \\
\hline $\mathrm{SiO}_{2}$ & 45.200 & 48.860 & 45.200 & 48.860 & 45.200 & 48.860 & 45.200 & 48.860 & 45.200 & 48.860 \\
\hline $\mathrm{TiO}_{2}$ & 0.200 & 0.370 & 0.200 & 0.370 & 0.200 & 0.370 & 0.200 & 0.370 & 0.200 & 0.370 \\
\hline $\mathrm{Al}_{2} \mathrm{O}_{3}$ & 3.940 & 17.720 & 3.940 & 17.720 & 3.940 & 17.720 & 3.940 & 17.720 & 3.940 & 17.720 \\
\hline $\mathrm{Fe}_{2} \mathrm{O}_{3}$ & 0.200 & 0.840 & 0.200 & 0.840 & 0.200 & 0.840 & 0.200 & 0.840 & 0.200 & 0.840 \\
\hline $\mathrm{Cr}_{2} \mathrm{O}_{3}$ & 0.400 & 0.030 & 0.400 & 0.030 & 0.400 & 0.030 & 0.400 & 0.030 & 0.400 & 0.030 \\
\hline $\mathrm{FeO}$ & 8.100 & 7.610 & 8.100 & 7.610 & 8.100 & 7.610 & 8.100 & 7.610 & 8.100 & 7.610 \\
\hline $\mathrm{MgO}$ & 38.400 & 9.100 & 38.400 & 9.100 & 38.400 & 9.100 & 38.400 & 9.100 & 38.400 & 9.100 \\
\hline $\mathrm{CaO}$ & 3.150 & 12.500 & 3.150 & 12.500 & 3.150 & 12.500 & 3.150 & 12.500 & 3.150 & 12.500 \\
\hline $\mathrm{Na}_{2} \mathrm{O}$ & 0.410 & 2.970 & 0.410 & 2.970 & 0.410 & 2.970 & 0.410 & 2.970 & 0.410 & 2.970 \\
\hline \multirow[t]{2}{*}{ sum } & 100 & 100 & 100 & 100 & 100 & 100 & 100 & 100 & 100 & 100 \\
\hline & \multicolumn{2}{|c|}{$\# 6(\mathrm{f}=5)$} & \multicolumn{2}{|c|}{$\# 7(\mathrm{f}=20)$} & \multicolumn{2}{|c|}{$\# 8(\mathrm{f}=100)$} & \multicolumn{2}{|c|}{$\# 9(\mathrm{f}=500)$} & \multicolumn{2}{|c|}{$\# 10(\mathrm{f}=1000)$} \\
\hline $\mathrm{SiO}_{2}$ & 45.200 & 48.860 & 45.200 & 48.860 & 45.200 & 48.860 & 45.200 & 48.860 & 45.200 & 48.860 \\
\hline $\mathrm{TiO}_{2}$ & 0.200 & 0.370 & 0.200 & 0.370 & 0.200 & 0.370 & 0.200 & 0.370 & 0.200 & 0.370 \\
\hline $\mathrm{Al}_{2} \mathrm{O}_{3}$ & 3.940 & 17.720 & 3.940 & 17.720 & 3.940 & 17.720 & 3.940 & 17.720 & 3.940 & 17.720 \\
\hline $\mathrm{Fe}_{2} \mathrm{O}_{3}$ & 0.200 & 0.840 & 0.200 & 0.840 & 0.200 & 0.840 & 0.200 & 0.840 & 0.200 & 0.840 \\
\hline $\mathrm{Cr}_{2} \mathrm{O}_{3}$ & 0.400 & 0.030 & 0.400 & 0.030 & 0.400 & 0.030 & 0.400 & 0.030 & 0.400 & 0.030 \\
\hline $\mathrm{FeO}$ & 8.100 & 7.610 & 8.100 & 7.610 & 8.100 & 7.610 & 8.100 & 7.610 & 8.100 & 7.610 \\
\hline $\mathrm{MgO}$ & 38.400 & 9.100 & 38.400 & 9.100 & 38.400 & 9.100 & 38.400 & 9.100 & 38.400 & 9.100 \\
\hline $\mathrm{CaO}$ & 3.150 & 12.500 & 3.150 & 12.500 & 3.150 & 12.500 & 3.150 & 12.500 & 3.150 & 12.500 \\
\hline $\mathrm{Na}_{2} \mathrm{O}$ & 0.410 & 2.970 & 0.410 & 2.970 & 0.410 & 2.970 & 0.410 & 2.970 & 0.410 & 2.970 \\
\hline \multirow[t]{2}{*}{ sum } & 100 & 100 & 100 & 100 & 100 & 100 & 100 & 100 & 100 & 100 \\
\hline & \multicolumn{2}{|c|}{$\# 11(\mathrm{f}=1)$} & \multicolumn{2}{|c|}{$\# 12(\mathrm{f}=1.5)$} & \multicolumn{2}{|c|}{$\# 13(\mathrm{f}=2)$} & \multicolumn{2}{|c|}{$\# 14(\mathrm{f}=5)$} & \multicolumn{2}{|c|}{$\# 15(\mathrm{f}=20)$} \\
\hline $\mathrm{SiO}_{2}$ & 47.434 & 48.860 & 47.434 & 48.860 & 47.434 & 48.860 & 47.434 & 48.860 & 47.434 & 48.860 \\
\hline $\mathrm{TiO}_{2}$ & 0.317 & 0.370 & 0.317 & 0.370 & 0.317 & 0.370 & 0.317 & 0.370 & 0.317 & 0.370 \\
\hline $\mathrm{Al}_{2} \mathrm{O}_{3}$ & 7.978 & 17.720 & 7.978 & 17.720 & 7.978 & 17.720 & 7.978 & 17.720 & 7.978 & 17.720 \\
\hline $\mathrm{Fe}_{2} \mathrm{O}_{3}$ & 0.582 & 0.840 & 0.582 & 0.840 & 0.582 & 0.840 & 0.582 & 0.840 & 0.582 & 0.840 \\
\hline $\mathrm{Cr}_{2} \mathrm{O}_{3}$ & 0.288 & 0.030 & 0.288 & 0.030 & 0.288 & 0.030 & 0.288 & 0.030 & 0.288 & 0.030 \\
\hline $\mathrm{FeO}$ & 7.595 & 7.610 & 7.595 & 7.610 & 7.595 & 7.610 & 7.595 & 7.610 & 7.595 & 7.610 \\
\hline $\mathrm{MgO}$ & 26.035 & 9.100 & 26.035 & 9.100 & 26.035 & 9.100 & 26.035 & 9.100 & 26.035 & 9.100 \\
\hline $\mathrm{CaO}$ & 7.902 & 12.500 & 7.902 & 12.500 & 7.902 & 12.500 & 7.902 & 12.500 & 7.902 & 12.500 \\
\hline $\mathrm{Na}_{2} \mathrm{O}$ & 1.869 & 2.970 & 1.869 & 2.970 & 1.869 & 2.970 & 1.869 & 2.970 & 1.869 & 2.970 \\
\hline \multirow[t]{2}{*}{ sum } & 100 & 100 & 100 & 100 & 100 & 100 & 100 & 100 & 100 & 100 \\
\hline & \multicolumn{2}{|c|}{$\# 16(\mathrm{f}=100)$} & \multicolumn{2}{|c|}{$\# 17(\mathrm{f}=500)$} & \multicolumn{2}{|c|}{$\# 18(\mathrm{f}=1.32)$} & $\overline{\# 1 c}$ & $\mathrm{f}=2)$ & $\# 20$ & $=5)$ \\
\hline $\mathrm{SiO}_{2}$ & 47.434 & 48.860 & 47.434 & 48.860 & 48.940 & 48.860 & 48.940 & 48.860 & 48.940 & 48.860 \\
\hline $\mathrm{TiO}_{2}$ & 0.317 & 0.370 & 0.317 & 0.370 & 0.393 & 0.370 & 0.393 & 0.370 & 0.393 & 0.370 \\
\hline $\mathrm{Al}_{2} \mathrm{O}_{3}$ & 7.978 & 17.720 & 7.978 & 17.720 & 10.394 & 17.720 & 10.394 & 17.720 & 10.394 & 17.720 \\
\hline $\mathrm{Fe}_{2} \mathrm{O}_{3}$ & 0.582 & 0.840 & 0.582 & 0.840 & 0.820 & 0.840 & 0.820 & 0.840 & 0.820 & 0.840 \\
\hline $\mathrm{Cr}_{2} \mathrm{O}_{3}$ & 0.288 & 0.030 & 0.288 & 0.030 & 0.237 & 0.030 & 0.237 & 0.030 & 0.237 & 0.030 \\
\hline $\mathrm{FeO}$ & 7.595 & 7.610 & 7.595 & 7.610 & 7.074 & 7.610 & 7.074 & 7.610 & 7.074 & 7.610 \\
\hline $\mathrm{MgO}$ & 26.035 & 9.100 & 26.035 & 9.100 & 18.887 & 9.100 & 18.887 & 9.100 & 18.887 & 9.100 \\
\hline $\mathrm{CaO}$ & 7.902 & 12.500 & 7.902 & 12.500 & 10.505 & 12.500 & 10.505 & 12.500 & 10.505 & 12.500 \\
\hline $\mathrm{Na}_{2} \mathrm{O}$ & 1.869 & 2.970 & 1.869 & 2.970 & 2.751 & 2.970 & 2.751 & 2.970 & 2.751 & 2.970 \\
\hline sum & 100 & 100 & 100 & 100 & 100 & 100 & 100 & 100 & 100 & 100 \\
\hline
\end{tabular}


Table S1. (continue) Initial bulk composition of the two assemblages and proportion factor $f$.

\begin{tabular}{|c|c|c|c|c|c|c|c|c|c|c|}
\hline bulk comp. & $\left(A_{0}\right)$ & $\left(B_{0}\right)$ & $\left(A_{0}\right)$ & $\left(B_{0}\right)$ & $\left(A_{0}\right)$ & $\left(B_{0}\right)$ & $\left(A_{0}\right)$ & $\left(B_{0}\right)$ & $\left(A_{0}\right)$ & $\left(B_{0}\right)$ \\
\hline oxides (g or wt $\%$ ) & \multicolumn{2}{|c|}{$\# 21(\mathrm{f}=20)$} & \multicolumn{2}{|c|}{$\# 22(\mathrm{f}=100)$} & \multicolumn{2}{|c|}{$\# 23(\mathrm{f}=500)$} & \multicolumn{2}{|c|}{$\# 24(\mathrm{f}=1)$} & \multicolumn{2}{|c|}{$\# 25(\mathrm{f}=10)$} \\
\hline $\mathrm{SiO}_{2}$ & 48.940 & 48.860 & 48.940 & 48.860 & 48.940 & 48.860 & 49.619 & 48.860 & 49.619 & 48.860 \\
\hline $\mathrm{TiO}_{2}$ & 0.393 & 0.370 & 0.393 & 0.370 & 0.393 & 0.370 & 0.426 & 0.370 & 0.426 & 0.370 \\
\hline $\mathrm{Al}_{2} \mathrm{O}_{3}$ & 10.394 & 17.720 & 10.394 & 17.720 & 10.394 & 17.720 & 11.372 & 17.720 & 11.372 & 17.720 \\
\hline $\mathrm{Fe}_{2} \mathrm{O}_{3}$ & 0.820 & 0.840 & 0.820 & 0.840 & 0.820 & 0.840 & 0.918 & 0.840 & 0.918 & 0.840 \\
\hline $\mathrm{Cr}_{2} \mathrm{O}_{3}$ & 0.237 & 0.030 & 0.237 & 0.030 & 0.237 & 0.030 & 0.219 & 0.030 & 0.219 & 0.030 \\
\hline $\mathrm{FeO}$ & 7.074 & 7.610 & 7.074 & 7.610 & 7.074 & 7.610 & 6.745 & 7.610 & 6.745 & 7.610 \\
\hline $\mathrm{MgO}$ & 18.887 & 9.100 & 18.887 & 9.100 & 18.887 & 9.100 & 16.074 & 9.100 & 16.074 & 9.100 \\
\hline $\mathrm{CaO}$ & 10.505 & 12.500 & 10.505 & 12.500 & 10.505 & 12.500 & 11.518 & 12.500 & 11.518 & 12.500 \\
\hline $\mathrm{Na}_{2} \mathrm{O}$ & 2.751 & 2.970 & 2.751 & 2.970 & 2.751 & 2.970 & 3.109 & 2.970 & 3.109 & 2.970 \\
\hline \multirow[t]{2}{*}{ sum } & 100 & 100 & 100 & 100 & 100 & 100 & 100 & 100 & 100 & 100 \\
\hline & \multicolumn{2}{|c|}{$\# 26(\mathrm{f}=20)$} & \multicolumn{2}{|c|}{$\# 27(\mathrm{f}=100)$} & \multicolumn{2}{|c|}{$\# 28(\mathrm{f}=500)$} & \multicolumn{2}{|c|}{$\# 29(\mathrm{f}=1)$} & \multicolumn{2}{|c|}{$\# 30(\mathrm{f}=5)$} \\
\hline $\mathrm{SiO}_{2}$ & 49.619 & 48.860 & 49.619 & 48.860 & 49.619 & 48.860 & 45.200 & 45.931 & 45.200 & 45.931 \\
\hline $\mathrm{TiO}_{2}$ & 0.426 & 0.370 & 0.426 & 0.370 & 0.426 & 0.370 & 0.200 & 0.199 & 0.200 & 0.199 \\
\hline $\mathrm{Al}_{2} \mathrm{O}_{3}$ & 11.372 & 17.720 & 11.372 & 17.720 & 11.372 & 17.720 & 3.940 & 18.599 & 3.940 & 18.599 \\
\hline $\mathrm{Fe}_{2} \mathrm{O}_{3}$ & 0.918 & 0.840 & 0.918 & 0.840 & 0.918 & 0.840 & 0.200 & 0.351 & 0.200 & 0.351 \\
\hline $\mathrm{Cr}_{2} \mathrm{O}_{3}$ & 0.219 & 0.030 & 0.219 & 0.030 & 0.219 & 0.030 & 0.400 & 0.015 & 0.400 & 0.015 \\
\hline $\mathrm{FeO}$ & 6.745 & 7.610 & 6.745 & 7.610 & 6.745 & 7.610 & 8.100 & 8.576 & 8.100 & 8.576 \\
\hline $\mathrm{MgO}$ & 16.074 & 9.100 & 16.074 & 9.100 & 16.074 & 9.100 & 38.400 & 17.507 & 38.400 & 17.507 \\
\hline $\mathrm{CaO}$ & 11.518 & 12.500 & 11.518 & 12.500 & 11.518 & 12.500 & 3.150 & 7.623 & 3.150 & 7.623 \\
\hline $\mathrm{Na}_{2} \mathrm{O}$ & 3.109 & 2.970 & 3.109 & 2.970 & 3.109 & 2.970 & 0.410 & 1.199 & 0.410 & 1.199 \\
\hline \multirow[t]{2}{*}{ sum } & 100 & 100 & 100 & 100 & 100 & 100 & 100 & 100 & 100 & 100 \\
\hline & \multicolumn{2}{|c|}{$\# 31(\mathrm{f}=20)$} & \multicolumn{2}{|c|}{$\# 32(\mathrm{f}=100)$} & \multicolumn{2}{|c|}{$\# 33(\mathrm{f}=500)$} & \multicolumn{2}{|c|}{$\# 34(\mathrm{f}=1)$} & \multicolumn{2}{|c|}{$\# 35(\mathrm{f}=5)$} \\
\hline $\mathrm{SiO}_{2}$ & 45.200 & 45.931 & 45.200 & 45.931 & 45.200 & 45.931 & 45.200 & 45.914 & 45.200 & 45.914 \\
\hline $\mathrm{TiO}_{2}$ & 0.200 & 0.199 & 0.200 & 0.199 & 0.200 & 0.199 & 0.200 & 0.216 & 0.200 & 0.216 \\
\hline $\mathrm{Al}_{2} \mathrm{O}_{3}$ & 3.940 & 18.599 & 3.940 & 18.599 & 3.940 & 18.599 & 3.940 & 18.582 & 3.940 & 18.582 \\
\hline $\mathrm{Fe}_{2} \mathrm{O}_{3}$ & 0.200 & 0.351 & 0.200 & 0.351 & 0.200 & 0.351 & 0.200 & 0.296 & 0.200 & 0.296 \\
\hline $\mathrm{Cr}_{2} \mathrm{O}_{3}$ & 0.400 & 0.015 & 0.400 & 0.015 & 0.400 & 0.015 & 0.400 & 0.005 & 0.400 & 0.005 \\
\hline $\mathrm{FeO}$ & 8.100 & 8.576 & 8.100 & 8.576 & 8.100 & 8.576 & 8.100 & 8.015 & 8.100 & 8.015 \\
\hline $\mathrm{MgO}$ & 38.400 & 17.507 & 38.400 & 17.507 & 38.400 & 17.507 & 38.400 & 18.551 & 38.400 & 18.551 \\
\hline $\mathrm{CaO}$ & 3.150 & 7.623 & 3.150 & 7.623 & 3.150 & 7.623 & 3.150 & 7.459 & 3.150 & 7.459 \\
\hline $\mathrm{Na}_{2} \mathrm{O}$ & 0.410 & 1.199 & 0.410 & 1.199 & 0.410 & 1.199 & 0.410 & 0.962 & 0.410 & 0.962 \\
\hline \multirow[t]{2}{*}{ sum } & 100 & 100 & 100 & 100 & 100 & 100 & 100 & 100 & 100 & 100 \\
\hline & \multicolumn{2}{|c|}{$\# 36(\mathrm{f}=20)$} & \multicolumn{2}{|c|}{$\# 37(\mathrm{f}=100)$} & \multicolumn{2}{|c|}{$\# 38(\mathrm{f}=500)$} & $\# 3$ & $=1)$ & $\# 40$ & $=5)$ \\
\hline $\mathrm{SiO}_{2}$ & 45.200 & 45.914 & 45.200 & 45.914 & 45.200 & 45.914 & 45.200 & 45.804 & 45.200 & 45.804 \\
\hline $\mathrm{TiO}_{2}$ & 0.200 & 0.216 & 0.200 & 0.216 & 0.200 & 0.216 & 0.200 & 0.281 & 0.200 & 0.281 \\
\hline $\mathrm{Al}_{2} \mathrm{O}_{3}$ & 3.940 & 18.582 & 3.940 & 18.582 & 3.940 & 18.582 & 3.940 & 18.319 & 3.940 & 18.319 \\
\hline $\mathrm{Fe}_{2} \mathrm{O}_{3}$ & 0.200 & 0.296 & 0.200 & 0.296 & 0.200 & 0.296 & 0.200 & 0.246 & 0.200 & 0.246 \\
\hline $\mathrm{Cr}_{2} \mathrm{O}_{3}$ & 0.400 & 0.005 & 0.400 & 0.005 & 0.400 & 0.005 & 0.400 & 0.015 & 0.400 & 0.015 \\
\hline $\mathrm{FeO}$ & 8.100 & 8.015 & 8.100 & 8.015 & 8.100 & 8.015 & 8.100 & 7.482 & 8.100 & 7.482 \\
\hline $\mathrm{MgO}$ & 38.400 & 18.551 & 38.400 & 18.551 & 38.400 & 18.551 & 38.400 & 18.834 & 38.400 & 18.834 \\
\hline $\mathrm{CaO}$ & 3.150 & 7.459 & 3.150 & 7.459 & 3.150 & 7.459 & 3.150 & 8.295 & 3.150 & 8.295 \\
\hline $\mathrm{Na}_{2} \mathrm{O}$ & 0.410 & 0.962 & 0.410 & 0.962 & 0.410 & 0.962 & 0.410 & 0.723 & 0.410 & 0.723 \\
\hline sum & 100 & 100 & 100 & 100 & 100 & 100 & 100 & 100 & 100 & 100 \\
\hline
\end{tabular}


Table S1. (continue) Initial bulk composition of the two assemblages and proportion factor $f$.

\begin{tabular}{|c|c|c|c|c|c|c|}
\hline bulk comp. & $\left(A_{0}\right)$ & $\left(B_{0}\right)$ & $\left(A_{0}\right)$ & $\left(B_{0}\right)$ & $\left(A_{0}\right)$ & $\left(B_{0}\right)$ \\
\hline oxides (g or wt $\%$ ) & \multicolumn{2}{|c|}{$41(\mathrm{f}=20)$} & \multicolumn{2}{|c|}{$42(\mathrm{f}=100)$} & \multicolumn{2}{|c|}{$43(f=500)$} \\
\hline $\mathrm{SiO}_{2}$ & 45.200 & 45.804 & 45.200 & 45.804 & 45.200 & 45.804 \\
\hline $\mathrm{TiO}_{2}$ & 0.200 & 0.281 & 0.200 & 0.281 & 0.200 & 0.281 \\
\hline $\mathrm{Al}_{2} \mathrm{O}_{3}$ & 3.940 & 18.319 & 3.940 & 18.319 & 3.940 & 18.319 \\
\hline $\mathrm{Fe}_{2} \mathrm{O}_{3}$ & 0.200 & 0.246 & 0.200 & 0.246 & 0.200 & 0.246 \\
\hline $\mathrm{Cr}_{2} \mathrm{O}_{3}$ & 0.400 & 0.015 & 0.400 & 0.015 & 0.400 & 0.015 \\
\hline $\mathrm{FeO}$ & 8.100 & 7.482 & 8.100 & 7.482 & 8.100 & 7.482 \\
\hline $\mathrm{MgO}$ & 38.400 & 18.834 & 38.400 & 18.834 & 38.400 & 18.834 \\
\hline $\mathrm{CaO}$ & 3.150 & 8.295 & 3.150 & 8.295 & 3.150 & 8.295 \\
\hline $\mathrm{Na}_{2} \mathrm{O}$ & 0.410 & 0.723 & 0.410 & 0.723 & 0.410 & 0.723 \\
\hline sum & 100 & 100 & 100 & 100 & 100 & 100 \\
\hline
\end{tabular}

